\title{
RENTABILIDADE E RISCO DE INVESTIMENTO NA PRODUÇÃO DE PALMITO DE PUPUNHA (Bactris gasipaes Kunth.)
}

\author{
Sidney Araujo Cordeiro ${ }^{1}$, Márcio Lopes da Silva²
}

(recebido: 17 de novembro de 2008; aceito: 27 de novembro de 2009)

\begin{abstract}
RESUMO: Neste estudo, objetivou-se realizar a análise financeira e uma simulação de risco de investimento em um projeto de produção de palmito de pupunha (Bactris gasipaes Kunth.). A análise financeira foi realizada mediante a aplicação dos métodos de avaliação de projetos: VPL, VAE, TIR e B/C; e para a análise de risco utilizou-se a técnica de simulação de Monte Carlo, mediante o programa @ RISK. Concluiu-se que o projeto de produção de palmito de pupunha é viável, com VPL de R\$ 3.549,08; TIR de 19\%; B/C maior que 1; e o VAE obtido foi de $\mathrm{R} \$ 1.049,12$. A análise de risco de investimento mostrou que o projeto apresenta uma probabilidade de $5 \%$ de se obter um valor negativo para o VPL. As variáveis que afetaram o VPL, na sua ordem de importância, foram: preço de venda do palmito, produtividade e taxa de juros.
\end{abstract}

Palavras-chave: Análise de risco, analise econômica, planejamento florestal.

\section{RENTABILITY AND INVESTMENT RISK IN PALM HEART OF PEJIBAYE (Bactris gasipaes Kunth.) CULTIVATION}

\begin{abstract}
This study carried out an economic study and a risk simulation analysis in a palm heart of pejibaye (Bactris gasipaes Kunth.) cultivation project. Economic analysis was made by using the project evaluation methods: NPV, VAE, TIR and B/C; and the risk analysis was carried out through the Monte Carlo simulation technique by @ RISK software. It was concluded that the project of palm heart of pejibaye cultivation is viable, with NPV of $R \$ 3.549,08$; TIR of 19\%; B/C greater than 1; the VAE of R\$1.049,12. The risk analysis showed that the project presents a probability of $5 \%$ of getting a negative value for VPL. The variables which affected Net Present Value (NPV) according to the ranking of importance were: selling price, productivity and discount rate.
\end{abstract}

Key words: Risk analysis, economic analysis, forest planning.

\section{INTRODUÇÃO}

A pupunha (Bactris gasipaes Kunth.) é uma palmeira originária da Região Amazônica, sendo domesticada e disseminada nesta região e na América Central por povos indígenas. É uma ótima alternativa para produção de palmito, podendo ser explorada em plantios organizados e possui características desejáveis, tais como precocidade, perfilhamento, rendimento e qualidade do seu palmito (CHAIMSOHN, 2000).

Bovi (2000) complementa, argumentando que o cultivo da pupunheira para produção de palmito vem despertando, desde a década de 70, o interesse de agricultores de todo o País. Esse interesse é decorrente, principalmente, da alta demanda, tanto interna quanto externa, de palmito de boa qualidade e à alta lucratividade da atividade. A busca de novas opções de cultivo em substituição às tradicionais, em virtude dos baixos preços alcançados por esses últimos no mercado, faz também com que empresários de outros setores se insiram no agronegócio do palmito de pupunha.

Em razão do potencial comercial do palmito de pupunha, muitos países latino-americanos estão investindo no seu cultivo e industrialização. O interesse para cultivar a pupunha está aumentando fortemente nos últimos anos (VILLACHICA, 1996), especialmente para a produção de palmito. Dois fatores que estão facilitando este crescimento: a existência de um mercado em nível mundial e a disponibilidade de tecnologia para o cultivo e industrialização da pupunha para palmito (VERRUMA-BERNARDI, 2007).

A tendência do mercado de palmito de pupunha é crescente, tanto em nível interno como internacionalmente, não só para atender as exigências do mercado internacional, como pela atual demanda dos frutos de açaí para produção da polpa, que apresenta maior rentabilidade que o mercado de palmito, apontando assim para uma diminuição paulatina deste

\footnotetext{
${ }^{1}$ Engenheiro Florestal, Doutorando em Ciência Florestal - Departamento de Engenharia Florestal/DEF - Universidade Federal de Viçosa/UFV - 36570-000 - Viçosa, MG - sidney.cordeiro@ufv.br

${ }^{2}$ Engenheiro Florestal, Professor Dr. em Ciência Florestal - Departamento de Engenharia Florestal/DEF - Universidade Federal de Viçosa/UFV - 36570-000 - Viçosa, MG - marlosil@ufv.br
}

Cerne, Lavras, v. 16, n. 1, p. 53-59, jan./mar. 2010 
palmito no mercado (Instituto Agronômico de Campinas IAC, 2008).

Diante do exposto, conduziu-se este trabalho, com o objetivo de verificar a rentabilidade da produção de palmito de pupunha, bem como realizar análise de risco de investimento para o projeto.

\section{MATERIAL E MÉTODOS}

\subsection{Aspectos técnicos de manejo da cultura}

De acordo com Mora Urpi (1999), a pupunheira, em virtude de estar associada a micorrizas, adapta-se a uma grande diversidade de solos, porém os melhores são aqueles profundos, bem drenados, textura areno argilosa, com topografia plana ou levemente ondulada, para facilitar a colheita e o transporte da produção. É oportuno ressaltar que em termos de solo, a textura e a drenagem são as variáveis mais importantes na implantação de um cultivo de pupunha, devendo ser evitados os solos mal drenados (a pupunheira não se desenvolve) e os solos compactados (prejudicam a emissão e o desenvolvimento dos perfilhos) e muito arenosos.

Apesar da adaptação a solos ácidos, para otimizar a eficiência dos nutrientes, o pH deve se manter entre 5,5 e 6. Quanto ao clima, tratando-se de uma espécie tropical, a região deve ter uma precipitação pluviométrica média anual acima de $1.600 \mathrm{~mm}$ bem distribuídos e temperatura média anual acima de $22^{\circ} \mathrm{C}$. Nas regiões de clima seco, é usada irrigação por aspersão, por canhão, pivô central, gotejamento e microaspersão. A escolha do sistema de irrigação é baseada não só na sua eficiência, como na disponibilidade de água e na relação custo-benefício (YUYAMA et al., 2005).

O plantio deve ser realizado em época de chuva, com mudas sadias e vigorosas, apresentando altura entre 30 a $40 \mathrm{~cm}$, com 5 a 6 folhas. A área deve estar limpa e balizada no espaçamento de $5 \times 5 \mathrm{~m}$ para produção de frutos, ou $2 \times 1 \mathrm{~m}(5.000 \mathrm{pl} / \mathrm{ha})$, para a produção de palmito (BERGO \& LUNZ, 2000).

\subsection{Análise financeira}

Para a análise financeira, considerou-se cultivos de pupunha com espaçamento de $2 \times 1 \mathrm{~m}$ no plantio da pupunha, obtendo-se uma densidade de 5.000 plantas/ha. O primeiro corte de pupunha foi realizado aos 2 anos de idade, o segundo aos 3 anos e o terceiro aos 4 anos.

Os custos e receitas envolvidos na produção do palmito de pupunha foram obtidos por meio de consultas a empresas do setor, no site do Cedagro (Centro de Desenvolvimento do Agronegócio), assim como com profissionais autônomos, no sentido de refletir a realidade da maioria dos plantios de pupunha com a finalidade de produção de palmito. Os custos são apresentados na Tabela 1.

Considerou-se uma produtividade de 2.000 hastes ou $800 \mathrm{~kg} / \mathrm{ha} \mathrm{no} 2^{\circ}$ ano e 4.500 hastes ou $2.250 \mathrm{~kg} /$ ha líquidos de palmito/ha a partir do $3^{\circ}$ ano.

Para fins da análise financeira e de viabilidade econômica, foi considerado o valor de comercialização do palmito de R\$2,50/peça. Salienta-se que este preço corresponde ao palmito comercializado em pé.

$\mathrm{Na}$ análise financeira, considerou-se uma taxa de juros de 6,75\% a.a., que é a taxa de juros de empréstimo de capital, inicialmente adotado pelo Programa de Plantio Comercial de Florestas (PROPFLORA) do Banco do Brasil e demais bancos credenciados pelo Banco Nacional de Desenvolvimento Econômico e Social (BNDES), para investimento e produção de florestas.

A análise financeira de todos os sistemas foi embasada nos métodos de avaliação de projetos apresentados e detalhados a seguir:

\section{Valor Presente Líquido - VPL}

O VPL representa a diferença entre as receitas e custos atualizados para uma determinada taxa de desconto (REZENDE \& OLIVEIRA, 2001; SILVA et al., 2002). Um VPL positivo indica que o projeto é economicamente viável, para uma determinada taxa utilizada. Na escolha entre dois ou mais projetos, deve-se selecionar o investimento com o maior VPL positivo.

$$
V P L=\sum_{j=1}^{n} \frac{R_{j}}{(1+i)^{j}}-\sum_{j=1}^{n} \frac{C_{j}}{(1+i)^{j}}
$$

Em que: $R_{j}=$ receitas no período $j ; C_{j}=$ custos no período $j ; i=$ taxa de desconto $; j=$ período de ocorrência de $R_{j}$ e $C_{j}$; e $n=$ duração do projeto, em anos, ou em número de períodos de tempo.

\section{Valor Anual Equivalente - VAE}

O Valor Anual Equivalente (VAE) é a parcela periódica e constante necessária ao pagamento de uma quantia igual ao VPL da opção de investimento em análise ao longo de sua vida útil. O projeto será considerado economicamente viável se o VAE for positivo. Assim, na identificação entre dois ou mais projetos, aquele com 
Tabela 1 - Custos médios de projetos de produção de palmito de pupunha.

Table 1 - Average costs of palm heart production.

\begin{tabular}{|c|c|c|c|c|c|c|c|c|c|c|}
\hline \multirow{3}{*}{ Itens de custo } & \multirow{3}{*}{ Unidade } & \multirow{3}{*}{$\begin{array}{c}\text { Valor } \\
\text { Unitário } \\
\text { (R\$/ha) }\end{array}$} & \multicolumn{8}{|c|}{ Período } \\
\hline & & & \multicolumn{2}{|c|}{$1^{\circ}$ ano } & \multicolumn{2}{|c|}{$2^{\circ}$ ano } & \multicolumn{2}{|c|}{$3^{\circ}$ ano } & \multicolumn{2}{|c|}{$4^{\circ}$ ano } \\
\hline & & & Quant & $(\mathrm{R} \$ / \mathrm{ha})$ & Quant & $(\mathrm{R} \$ / \mathrm{ha})$ & Quant & $(\mathrm{R} \$ / \mathrm{ha})$ & Quant & $(\mathrm{R} \$ / \mathrm{ha})$ \\
\hline \multicolumn{11}{|l|}{$\begin{array}{l}\text { 1. INSUMOS E } \\
\text { EQUIPAMENTOS }\end{array}$} \\
\hline Mudas (Plantio e Replantio) & ud & 1,5 & 5.500 & $8.250,00$ & & & & & & \\
\hline Calcário & $\mathrm{t}$ & 89,67 & 1,5 & 134,51 & & & 2,00 & 179,34 & 2,00 & 179,34 \\
\hline Nitrogênio - N & $\mathrm{kg}$ & 2,95 & 110 & 324,50 & 180,00 & 531,00 & 250,00 & 737,50 & 250,00 & 737,50 \\
\hline Fósforo $-\mathrm{P}_{2} \mathrm{O}_{5}$ & $\mathrm{~kg}$ & 2,52 & 50 & 126,00 & & & & & & \\
\hline Potássio - $\mathrm{K}_{2} \mathrm{O}$ & $\mathrm{kg}$ & 1,26 & 50 & 63,00 & 75,00 & 94,50 & 100,00 & 126,00 & 100,00 & 126,00 \\
\hline Esterco de Galinha & $\mathrm{T}$ & 100 & 7,5 & 750,00 & & & & & & \\
\hline Energia Elétrica & $\mathrm{kw} / \mathrm{h}$ & 0,20 & 1500 & 300,00 & $1.500,00$ & 300,00 & $1.500,00$ & 300,00 & $1.500,00$ & 300,00 \\
\hline $\begin{array}{l}\text { Conjunto de irrigação por } \\
\text { aspersão* }\end{array}$ & ud & $1.955,00$ & 1 & $1.955,00$ & & & & & & \\
\hline Subtotal & $\mathrm{R} \$$ & & & $11.903,01$ & & 925,50 & & $1.342,84$ & & $1.342,84$ \\
\hline \multicolumn{11}{|l|}{ 2. SER VIÇOS } \\
\hline Aração & $\mathrm{h} / \mathrm{M}$ & 50,00 & 3 & 150,00 & & & & & & \\
\hline Gradagem & $\mathrm{h} / \mathrm{M}$ & 50,00 & 2 & 100,00 & & & & & & \\
\hline M arcação de cova & $\mathrm{d} / \mathrm{H}$ & 20,00 & 7 & 140,00 & & & & & & \\
\hline Coveamento & $\mathrm{d} / \mathrm{H}$ & 20,00 & 22 & 440,00 & & & & & & \\
\hline Calagem & $\mathrm{d} / \mathrm{H}$ & 20,00 & 4 & 80,00 & & & 4 & 80,00 & 4 & 80,00 \\
\hline Adubação nas covas & $\mathrm{d} / \mathrm{H}$ & 20,00 & 6 & 120,00 & & & & & & \\
\hline Plantio e Replantio & $\mathrm{d} / \mathrm{H}$ & 20,00 & 16 & 320,00 & & & & & & \\
\hline Capina manual na linhas & $\mathrm{d} / \mathrm{H}$ & 20,00 & 8 & 160,00 & & & & & & \\
\hline $\begin{array}{l}\text { Roçagem manual nas } \\
\text { entrelinhas }\end{array}$ & $\mathrm{d} / \mathrm{H}$ & 20,00 & 3 & 60,00 & 4 & 80,00 & 4 & 80,00 & 4 & 80,00 \\
\hline Manejo de irrigação & $\mathrm{d} / \mathrm{H}$ & 20,00 & 20 & 400,00 & 20 & 400,00 & 20 & 400,00 & 20 & 400,00 \\
\hline Adubação de Cobertura & $\mathrm{d} / \mathrm{H}$ & 20,00 & 5 & 100,00 & 8 & 160,00 & 10 & 200,00 & 10 & 200,00 \\
\hline Colheita manual & $\mathrm{d} / \mathrm{H}$ & 20,00 & & & 11 & 220,00 & 25 & 500,00 & 25 & 500,00 \\
\hline Subtotal & $\mathrm{R} \$$ & & & $2.070,00$ & & 860,00 & & $1.260,00$ & & $1.260,00$ \\
\hline TOTAL & $\mathrm{R} \$$ & & & $13.973,01$ & & $1.785,50$ & & $2.602,84$ & & $2.602,84$ \\
\hline
\end{tabular}

* Possui um valor final de $20 \%$ e vida útil de 10 anos.

Fonte: Adaptado de Centro de Desenvolvimento do Agronegócio - CEDAGRO (2008) e empresas do setor.

melhor condição de ser executado é o projeto que apresentar o maior VAE (REZENDE \& OLIVEIRA, 2001; SILVA et al., 2002).

$$
V A E=\frac{V P L \cdot i}{1-(1+i)^{-n}}
$$

Em que: $V P L=$ valor presente líquido; $n=$ duração do ciclo ou rotação, em anos.

\section{Taxa Interna de Retorno - TIR}

A TIR é a taxa de desconto que iguala o valor atual das receitas futuras ao valor atual dos custos futuros do projeto. Assim sendo, esta técnica se constitui uma medida relativa que reflete o aumento no valor do investimento ao longo do tempo, com base nos recursos requeridos para produzir o fluxo de receitas (REZENDE \& OLIVEIRA, 2001; SILVA et al., 2002). 


$$
\sum_{j=1}^{n} \frac{R_{j}}{(1+T I R)^{j}}-\sum_{j=1}^{n} \frac{C_{j}}{(1+T I R)^{j}}=0
$$

Em que: $T I R=$ taxa interna de retorno; as demais variáveis já foram definidas.

\section{Razão Benefício/Custo - B/C}

Este método consiste em determinar a relação entre o valor presente dos benefícios e o valor presente dos custos, para uma determinada taxa de juros ou descontos. Um projeto é considerado viável economicamente se $B / C>1$. Entre dois ou mais projetos, o mais viável é aquele que apresentar o maior valor de $B / C$ (REZENDE \& OLIVEIRA, 2001). Quando $B / C=1$, resulta em $V P L=0$; nesse caso, a $T I R$ associada a um projeto pode também ser determinada como sendo a taxa que faz com que $B / C=1$.

$$
B / C=\frac{\sum_{j=0}^{n} R_{j}(1+i)^{-j}}{\sum_{j=0}^{n} C_{j}(1+i)^{-j}}
$$

Em que: $R_{j}=$ receita no final do ano $j ; C_{i}=$ custo no final do ano $j$; e $n=$ duração do projeto, em anos.

\subsection{Análise de risco}

De maneira geral, os benefícios e custos associados ao fluxo de caixa de projetos de investimento são, normalmente, considerados conhecidos, caracterizando o que se conhece como procedimento de análise determinística; que, apesar de sua praticidade, leva a uma simplificação e, ou, a superestimativa de informações que nem sempre são conhecidas com certeza no momento da análise, como preços, quantidades, e rendimentos, entre outros. Uma forma de minimizar esse problema é adotar uma análise em condições de risco, em que se utilizam distribuições de probabilidade associadas aos indicadores de desempenho do projeto (BENTESGAMA et al., 2005).
$\mathrm{Na}$ presente análise de risco de investimento, utilizaram-se as informações do projeto em estudo na análise financeira. Os dados foram analisados mediante o software @ RISK (PALISADE CORPORATION, 1995). Esse programa permite a aplicação do método de Monte Carlo para simular valores para as variáveis aleatórias receita e custo e, em decorrência dos valores aleatórios gerados, obter valores para a variável lucro (BENTES-GAMA, 2003). Foram definidas 10.000 interações e consideradas como variáveis de entrada (inputs): taxa anual de juros; preço do palmito (unidade); produtividade de palmito por hectare. Consideraram-se ainda variações entre $-20 \% a+20 \%$ nessas variáveis com base na distribuição triangular, adotado por Bentes-Gama (2003). O indicador financeiro VPL foi tomado como variável de saída (output).

\section{RESULTADOS E DISCUSSÃO}

\subsection{Análise financeira}

O fluxo de caixa para o projeto está apresentado na Tabela 2, sendo construído anualmente, visando a um horizonte de planejamento de 4 anos.

Os resultados obtidos por meio dos diferentes métodos de análise financeira estão apresentados na Tabela 3.

O VPL do projeto analisado foi maior do que zero, significando que é viável economicamente, sendo de $\mathrm{R} \$ 3.549,08$. Vale lembrar que o VPL representa o lucro do negócio corrigido pela taxa de juros - para um horizonte de 4 anos.

A TIR é a taxa que representa o retorno financeiro do projeto. A TIR apresentada foi de 19\%. A TIR obtida foi maior do que a taxa de desconto, que, no caso, é de $6,75 \%$ ao ano, o que significa que o projeto é viável, sob este ponto de vista.

Em pesquisa realizada por Sampaio et al. (2007), quando foram comparadas produções de palmito de

Tabela 2 - Fluxo de caixa para o projeto de produção de palmito de pupunha.

Table 2 - Cash flow for a palm heart project using pejibaye cultivation.

\begin{tabular}{cccc}
\hline \multicolumn{3}{c}{ Produção de palmito } \\
\hline Tempo (ano) & Custos (R $\$$ /ha) & Receitas (R \$/ha) & Saldo (R\$/ha) \\
\hline 1 & $13.973,01$ & 0,00 & $-13.973,01$ \\
3 & $1.785,50$ & $5.000,00$ & $3.214,50$ \\
4 & $2.602,84$ & $11.250,00$ & $8.647,16$ \\
\end{tabular}

Cerne, Lavras, v. 16, n. 1, p. 53-59, jan./mar. 2010 
pupunha e palmeira-real, observou-se que ambas são viáveis economicamente, sendo que a produção de palmito de pupunha obteve TIR de 53,27\%. Essa diferença é decorrente da produtividade que, na pesquisa citada, foi de 13,5 t/ha/ano de palmito de pupunha e, na presente pesquisa, variou de 2 a 4,5t/ha/ano. O Instituto Agronômico de Campinas (IAC, 1998) menciona que a produtividade de palmito de pupunha esperada é de 2,6 a 3,6 t/ha, porém, considerando o corte aos 18 meses de idade.

Tabela 3 - Análise financeira para o projeto de produção de palmito de pupunha.

Table 3 - Financial analysis for palm heart project using of pejibaye cultivation.

\begin{tabular}{cc}
\hline Métodos de avaliação & Valores \\
\hline VPL (R\$/ha) & $3.594,08$ \\
TIR (\% a. a.) & 19,00 \\
VAE (R/ha/ano) & $1.049,12$ \\
B/C & 1,19 \\
\hline
\end{tabular}

O projeto é viável, uma vez que o VAE se apresentou positivo, e que representa o lucro anual do negócio. De acordo com a Tabela 3, o lucro anual foi de R\$1.049,12.

A razão $\mathrm{B} / \mathrm{C}$ foi maior que 1 , indicando também que o projeto é viável economicamente. Essa razão foi de 1,19, o que significa que as receitas superam os custos em 19\%. Essa razão B/C encontrada foi inferior à encontrada por Sá et al. (2002), em estudo realizado no Acre, onde foi observado o valor de 1,31, a uma taxa de desconto de $6 \%$.

\subsection{Análise de risco de investimento}

Mediante as simulações realizadas pelo @ RISK, foram obtidos os resultados financeiros e suas respectivas probabilidades acumuladas para o projeto em estudo. O valor médio do VPL foi de R $\$ 3.650,46 / \mathrm{ha} /$ ano (Tabela 4).

Considerando os resultados da simulação gerados para o VPL do projeto, a análise de percentis indicou que $5 \%$ dos valores estão acima de R \$ 7.684,99/ha/ano e que $5 \%$ dos valores são negativos. Assim sendo, considerando

Tabela 4 - Estatísticas da variável de saída (VPL) e de entrada (taxa de desconto, preço de venda e produtividade) do projeto.

Table 4 - Statistics of output (NPV) and input (discount rate, selling price and productivity) variables of the project.

\begin{tabular}{|c|c|c|c|c|}
\hline \multirow{2}{*}{ Estatísticas } & \multicolumn{3}{|c|}{ Variáveis de entrada } & \multirow{2}{*}{$\begin{array}{c}\text { Variável de saída } \\
\text { VPL (R\$/ha) }\end{array}$} \\
\hline & $\mathrm{Tj}$ & PV & $\mathrm{Pd}$ & \\
\hline Mínimo & 5,21 & 2,00 & $3.607,07$ & $-3.141,06$ \\
\hline Máximo & 7,79 & 2,99 & $5.392,31$ & $12.050,78$ \\
\hline Média & 6,49 & 2,49 & $4.504,38$ & $3.650,46$ \\
\hline Desvio-padrão & 0,53 & 0,21 & 370,547 & $2.388,84$ \\
\hline Moda & 6,48 & 2,51 & $4.244,69$ & $2.334,84$ \\
\hline \multicolumn{5}{|c|}{ Percentis } \\
\hline $5 \%$ & 0,056 & 2,15 & $3.889,88$ & $-155,41$ \\
\hline $15 \%$ & 0,059 & 2,27 & $4.001,90$ & $1.088,49$ \\
\hline $25 \%$ & 0,061 & 2,34 & $4.089,56$ & $1.942,49$ \\
\hline $35 \%$ & 0,063 & 2,41 & $4.169,28$ & $2.614,61$ \\
\hline $45 \%$ & 0,064 & 2,47 & $4.234,46$ & $3.242,95$ \\
\hline $55 \%$ & 0,066 & 2,52 & $4.296,48$ & $3.875,61$ \\
\hline $65 \%$ & 0,067 & 2,58 & $4.353,06$ & $4.549,09$ \\
\hline $75 \%$ & 0,069 & 2,64 & $4.405,92$ & $5.333,76$ \\
\hline $85 \%$ & 0,071 & 2,72 & $4.456,60$ & $6.219,48$ \\
\hline $95 \%$ & 0,074 & 2,84 & $4.501,16$ & $7.684,99$ \\
\hline
\end{tabular}

VPL: valor presente líquido (R $\$$ ha); Tj: taxa anual de juros (\%); Pv: preço de venda do palmito; Pd: produtividade (kg/ha/ano). 
que sejam mantidas todas as condições de estabilidade de mercado ao longo do projeto, pode-se afirmar que esse projeto apresenta elevada viabilidade econômica e baixo risco de investimento (Tabela 4). Como complementação da análise, a função densidade de probabilidade simulada para o projeto que determina com precisão um intervalo de confiança, no qual venham a ocorrer os valores desejados ou procurados (BENTES-GAMA, 2005), confirma que a probabilidade de ocorrência de VPL negativo é de $5 \%$.

Na Figura 1, são apresentados os valores dos coeficientes de elasticidade para as variáveis de preço, produtividade e taxa de juros.

Observando-se a Figura 1, de acordo com a análise, os valores positivos das elasticidades indicaram uma relação direta entre as referidas variáveis, ocorrendo efeito inverso quando elas apresentaram valores negativos. Analisando, por exemplo, as variações para o VPL do projeto, pode-se interpretar que, caso ocorra um aumento de $10 \%$ no preço de venda do palmito, haverá um aumento de $7,78 \%$ sobre o seu valor. Por outro lado, um aumento de $10 \%$ na taxa de juros poderá ocasionar uma diminuição de $0,8 \%$ no VPL. Observa-se também, que um aumento de $10 \%$ na produtividade ocasionará um aumento de $6,24 \%$ sobre o valor do VPL.

As variáveis que afetaram o VPL, na sua ordem de importância, foram: preço de venda do palmito, produtividade e taxa de juros. Sendo assim, o preço de

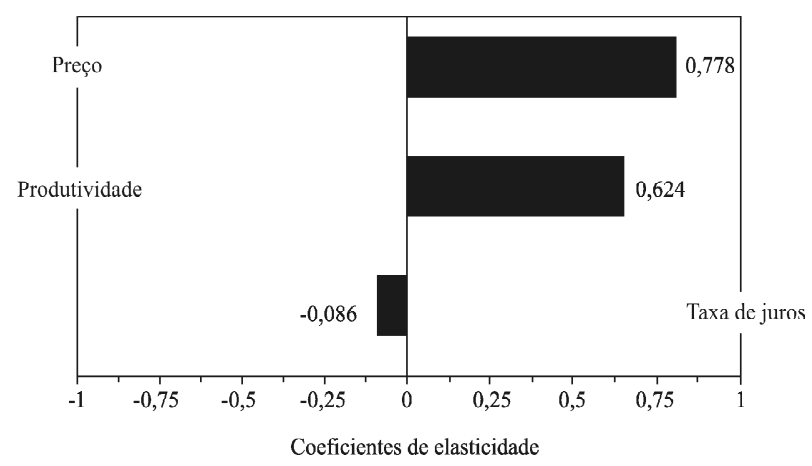

Figura 1 - Análise de sensibilidade com base nas elasticidades das variáveis de entrada (taxa de desconto, preço de venda e produtividade), de saída (VPL) e ordem de influência na análise do projeto.

Figure 1 - Sensibility analyses based on the elasticity of the input (discount rate, vend price and productivity), the output $(N P V)$ variables, and the influence order on the analysis of the project.

Cerne, Lavras, v. 16, n. 1, p. 53-59, jan./mar. 2010 venda do palmito é mais influente de forma negativa sobre o VPL do projeto (Figura 1).

\section{CONCLUSÕES}

O projeto de produção de palmito de pupunha apresenta viabilidade econômica de acordo com os métodos utilizados.

A análise de risco de investimento mostrou que o projeto apresenta uma probabilidade de $5 \%$ de se obter um valor negativo para o VPL.

As variáveis que afetaram o VPL, na sua ordem de importância, foram: preço de venda do palmito, produtividade e taxa de juros.

\section{AGRADECIMENTOS}

Ao Conselho Nacional de Desenvolvimento Científico e Tecnológico (CNPQ) pelo apoio financeiro e à Universidade Federal de Viçosa pelo fornecimento da estrutura e pessoal.

\section{REFERÊNCIAS BIBLIOGRÁFICAS}

BENTES-GAMA, M. M. de. Análise técnica e econômica de sistemas agroflorestais em Machadinho d'Oeste, Rondônia. 2003. 112 f. Tese (Doutorado em Ciência Florestal) - Universidade Federal de Viçosa, Viçosa, 2003.

BENTES-GAMA, M. M. de et al. Análise econômica de sistemas agroflorestais na Amazônia Ocidental, Machadinho D'Oeste RO. Revista Árvore, Viçosa, v. 29, n. 3, p. 401-411, 2005.

BERGO, C. L.; LUNZ, A. M. P. Cultivo da pupunha para palmito no Acre. Rio Branco: Embrapa Acre, 2000. 15 p. (Embrapa Acre. Circular Técnica, 31).

BOVI, M. L. A. O agronegócio palmito de pupunha. O Agronômico, Campinas, v. 52, n. 1, 2000.

CENTRO DE DESENVOLVIMENTO DO AGRONEGÓCIO. Disponível em: 'khttp://Www.cedagro.org.bry. Acesso em: 26 set. 2008.

CHAIMSOHN, F. P. Cultivo de pupunha e produção de palmito. Viçosa, MG: Aprenda Fácil, 2000. 121 p.

InStituto AGRONÔMICO DE CAMPINAS. Pupunha (Bactris gasipaes Kunth). Disponível em: <http:// 'www.iac.sp.gov.br'. Acesso em: 15 jul. 2008. 
MORA URPI, J. Palmito de Pejibaye (Bactris gasipaes

Kunth): su cultivo e industrialización. San José: Universidad de

Costa Rica, 1999. 31 p.

PALISADE CORPORATION. Risk analysis and simulation add-in for Microsoft Excell or Lotus 1-2-3. New York: Palisade Corporation, 1995. 402 p.

REZENDE, J. L. P.; OLIVEIRA, A. D. Análise econômica e social de projetos florestais. Viçosa, MG: UFV, 2001. 389 p.

SÁ, C. P.; BERGO, C. L.; SANTOS, J. C.; NASCIMENTO, G. C.; GOMES, F. C. R. Coeficientes técnicos e avaliação econômica para o sistema de produção melhorado da pupunha para produção de palmito no Acre. Rio Branco: Embrapa Acre, 2002. (Comunicado Técnico, 150).

SAMPAIO, L. C.; LELIS, P. S. S.; SILVA, J. A.; VILLA, E. B. Análise técnica e econômica da produção de palmito de pupunha
(Bactris gasipaes Kunth.) e de palmeira-real (Archontophoenix alexandrae Wendl. \& Drude). Floresta e Ambiente, Seropédica, v. 14, n. 1, p. 14-24, 2007.

SILVA, M. L.; JACOVINE, L. A. G.; VALVERDE, S. R. Economia florestal. Viçosa, MG: UFV, 2002. 178 p.

VERRUMA-BERNARDI, M. R.; MORAES, C. W. S. de; MACHADO, C. A.; KAJISHIMA, S.; COSTA, E. Q. Análise descritiva quantitativa do palmito de pupunheira. Acta Amazonica, Manaus, v. 37, n. 4, 2007.

VILLACHICA, H. Cultivo del pijuayo (Bactris gasipaes Kunth.) para palmito en la Amazonia. Lima: FAO, 1996. 153 p.

YUYAMA, K.; CHÁVEZ, W. B.; PEREIRA, B. G.; SILVA, I. A. Efeito da densidade de plantas e da adubação NPK na produção inicial de palmito de pupunheira. Revista Brasileira de Ciência do Solo, Viçosa, v. 29, p. 373-378, 2005. 